\title{
Meningioma na tenda cerebelar: diagnóstico e tratamento
}

\author{
Meningioma on the cerebellar tent: diagnosis and treatment \\ Meningioma de la tienda del cerebelo: diagnóstico y tratamiento \\ Carolina Ruiz MAZAIA ${ }^{1}$ \\ Caroline Sanches Vick FRANCISCO${ }^{1}$ \\ Deolino João CAMILO JUNIOR ${ }^{2}$ \\ Luciana Estevam SIMONATO \\ Gabriel Pina PAIVA ${ }^{3}$ \\ ${ }^{1}$ Graduanda do Curso de Medicina da Universidade Brasil, 15600-000 Fernandópolis - SP, Brasil \\ ${ }^{2}$ Patologista do IPAT - Instituto de Patologia de Araçatuba, 16015-000 Araçatuba - SP, Brasil \\ ${ }^{3}$ Docentes do Curso de Medicina da Universidade Brasil, 15600-000 Fernandópolis - SP, Brasil
}

\begin{abstract}
Resumo
O presente trabalho relatou um caso clínico de meningioma na tenda cerebelar, evidenciando seus aspectos clínicos, imaginológicos e histopatológicos, com o intuito de ampliar o conhecimento do médico generalista, favorecendo o diagnóstico e o tratamento da doença. O caso relatado foi de um paciente do sexo masculino na sétima década de vida, que permaneceu com a lesão durante 4 meses após apresentar os primeiros sinais e sintomas. O diagnóstico definitivo foi realizado com auxílio de ressonância magnética e, imediatamente, a opção terapêutica instituída foi a ressecção completa da lesão. Mediante tal relato, pode-se concluir que a investigação clínica é essencial mesmo em pacientes fora do grupo de maior incidência da lesão, sendo que o conhecimento das principais características da doença facilita o diagnóstico precoce e, consequentemente, antecipa o tratamento adequado, resultando no melhor prognóstico do paciente.
\end{abstract}

Descritores: Meningioma; Neoplasias Meníngeas; Neoplasias de Tecido Nervoso.

\begin{abstract}
The present study reported a clinical case of meningioma in the cerebellar tent, evidencing its clinical, imaging and histopathological aspects, in order to broaden the knowledge of the general practitioner, favoring the diagnosis and treatment of the disease. The case reported was of a male patient in the seventh decade of life, who remained with the lesion for 4 months after presenting the first signs and symptoms. The definitive diagnosis was made with the aid of magnetic resonance imaging, and immediately, the therapeutic option instituted was the complete resection of the lesion. By means of such a report, it can be concluded that clinical investigation is essential even in patients outside the group with a higher incidence of the injury, and knowledge of the main characteristics of the disease facilitates early diagnosis and, consequently, anticipates appropriate treatment, resulting in better prognosis for patients.

Descriptors: Meningioma; Meningeal Neoplasms; Neoplasms, Nerve Tissue.
\end{abstract}

\section{Resumen}

Este estudio informó un caso de meningioma en la tienda del cerebelo, mostrando su clínica, las imágenes y los aspectos histopatológicos, con el fin de ampliar los conocimientos de la medicina general, favoreciendo el diagnóstico y tratamiento de la enfermedad. Presentamos el caso de un paciente varón de en la séptima década de la vida, que se quedó con la lesión durante 4 meses después de presentar los primeros signos y síntomas. El diagnóstico final se hizo con la ayuda de resonancia magnética y de inmediato la opción de terapia era una resección completa de la lesión. A tal cuenta, se puede concluir que la investigación clínica es esencial incluso en pacientes más allá de la mayor incidencia de grupo lesión, con el conocimiento de las características principales de la enfermedad facilita el diagnóstico temprano y, por consiguiente, se anticipa el tratamiento apropiado, lo que resulta en mejor pronóstico.

Descriptores: Meningioma; Neoplasias Meníngeas; Neoplasias de Tejido Nervioso.

\section{INTRODUÇÃO}

O meningioma é uma neoplasia benigna localizada na membrana meníngea ou nas vilosidades aracnóideas. Para Pimentel $^{1}$ a vilosidade aracnóidea é constituída por uma cápsula fibrosa com uma camada de células aracnóideas em seu interior que se comunica com o seio venoso através de uma estrutura trabecular.

Segundo Velasquez et al. $^{2}$ as células aracnóideas possuem alto risco de desenvolver neoplasias, caracterizadas pelo crescimento anormal de células devido a danos em regiões do DNA que controlam a proliferação celular; metaplasias, pelo aumento de células neoplásicas gerando disfunções metabólicas e perda da função do órgão; ou estarem associadas a metástases devido ao seu contato com o seio venoso.

Os meningiomas correspondem a cerca de $20 \%$ dos tumores intracranianos, sendo o segundo tumor intracraniano primário mais comum e com ocorrência maior em mulheres (2:1) entre a quarta e quinta década de vida, de acordo com Costa Jr. et al. ${ }^{3}$. Esse estudo também afirmou que a lesão tem origem nas mutações das células meningoteliais da aracnóide e que, a maioria, é histologicamente benigna com prognóstico dependente da acessibilidade e ressecabilidade do tumor.

Com relação aos tumores intracranianos cabe ressaltar que, segundo Cambruzzi et al. ${ }^{4}$, o cérebro e o cerebelo são os locais mais comuns de metástases (aproximadamente $75 \%)$, sendo os pulmões $(22,36 \%)$, os rins $(16,21 \%)$, a mama $(14,85 \%)$ e o cólon $(8,1 \%)$ os sítios primários mais frequentes. No encéfalo, as metástases podem ser encontradas nas meninges (cerca de 10 a 15\%) ou sob a forma intraparenquimatosa (em torno de $90 \%$ ), sendo que os sítios primários mais comuns em adultos, em ordem 
decrescente, são: pulmão (carcinoma de pequenas células e adenocarcinoma), mama (carcinoma ductalinfiltrante), rim (carcinoma de células claras) e intestino grosso (adenocarcinoma).

O diagnóstico dos tumores intracranianos pode ser obtido por meio de diversos métodos como descrito por Pimentel ${ }^{1}$. Em seu estudo mostrou que na tomografia computadorizada (TC) é possível verificar características tomodensiométricas de meningiomas benignos, enquanto que na ressonância magnética nuclear (RM) verifica-se com maior detalhe a topografia, as dimensões e as recidivas pósoperatórias.

A manifestação clínica mais frequente nos casos de meningioma de acordo com Lima et al. ${ }^{5}$ foi a cefaleia, o que pode estar associado ao diagnóstico tardio dos pacientes. Outra queixa em elevado número de pacientes é o vômito. Ambos os sintomas podem estar associados à hipertensão intracraniana em quadros clínicos avançados.

$\mathrm{O}$ estudo de Castro et al. ${ }^{6}$ mostrou que a imagem de lesões intracranianas de etiologia tumoral tem características próprias e apresenta padrões de imagem que facilitam o diagnóstico. No entanto, a definição do diagnóstico pode ser dificultada se houver o desconhecimento dos tipos de tecido que podem dar origem ao tumor. De acordo com os autores, a TC mostra, normalmente, uma massa lobulada hiperdensa nitidamente demarcada, podendo haver a presença de edema. Também podem ocorrer calcificações com contraste significativo, segundo Valenzuela, Ebensperguer ${ }^{7}$ e no qual Castro et al. ${ }^{6}$ complementaram dizendo que ocorrem em $50 \%$ dos casos. Já na RM o tumor pode aparecer variando de iso a hipointenso em $\mathrm{T} 1 \mathrm{e}$, em geral, isointenso em $\mathrm{T} 2$, com intenso realce pelo meio de contraste, como descreve Shogan et al. ${ }^{8}$

As características histológicas relatadas como típicas dos meningiomas são interdigitações das membranas celulares, presença de material filamentoso intracitoplasmático e junções desmossômicas. Além disso, as lesões são acompanhadas da presença de edema vasogênico cerebral, segundo estudos de Pimentel ${ }^{1}$.

De acordo com Jesús et al. ${ }^{9}$ a terapêutica para os meningiomas depende dos fatores relacionados ao doente e ao tumor, pois visa proporcionar sobrevida, manter ou melhorar sintomas neurológicos e a qualidade de vida do paciente. Há diversas opções de tratamento: observação, ressecção cirúrgica, radioterapia, quimioterapia ou combinações destas técnicas.

Caso o paciente seja assintomático, sendo diagnosticado incidentalmente, pode-se optar pela observação, desde que haja o acompanhamento baseado no histórico, exames clínicos e avaliação periódica por TC e RM, de acordo com Pamir et al. ${ }^{10}$.

No tratamento neurocirúrgico a magnitude da ressecção e a possibilidade de ressecção completa, são fatores importantes para o prognóstico e o aumento da sobrevida do paciente, segundo Al-Mefty et al. ${ }^{11}$.

Ainda de acordo com Jesús et al. ${ }^{9}$ quando a ressecção cirúrgica é incompleta, opta-se por radioterapia como tratamento adjuvante.

Sacko et al. ${ }^{12}$ destacaram que os fatores relacionados à mortalidade operatória são: idade avançada, desenvolvimentos intracranianos, hipertensão intracraniana, ressecção incompleta da lesão e más condições clínicas préoperatórias. O propósito do presente trabalho é relatar um caso clínico de meningioma na tenda cerebelar, evidenciando seus aspectos clínicos, imaginológicos e histopatológicos, com o intuito de ampliar o conhecimento do médico generalista, favorecendo o diagnóstico e o tratamento da doença.

\section{CASO CLÍNICO}

Paciente do sexo masculino, leucoderma, com 74 anos de idade, aposentado, casado, natural de Braúna-SP, procurou atendimento médico com queixa de perda progressiva dos movimentos dos membros inferiores há 8 meses. Devido à dificuldade em andar e se manter de pé, primeiramente, procurou um ortopedista. O profissional realizou infiltração, onde o paciente se queixava de dor intensa e prescreveu sessões de fisioterapia. No entanto, como não houve melhora dos sinais e sintomas o mesmo foi encaminhado para um neurologista.

A princípio a perda da mobilidade das pernas foi associada, pelo neurologista, à doença de Alzheimer, pois o paciente apresentava tônus muscular, mas sem coordenação motora e equilíbrio. Foi realizada RM e o resultado negou a hipótese diagnóstica, pois foi verificada formação nodular homogênea localizada junto à tenda cerebelar à direita, com isosinal na ponderação $\mathrm{T} 1 \mathrm{e}$ impregnação difusa e homogênea após infusão do contraste ( 2.9 x 2.5 x 2.4), como pode ser observado nas Figuras 1 e 2.

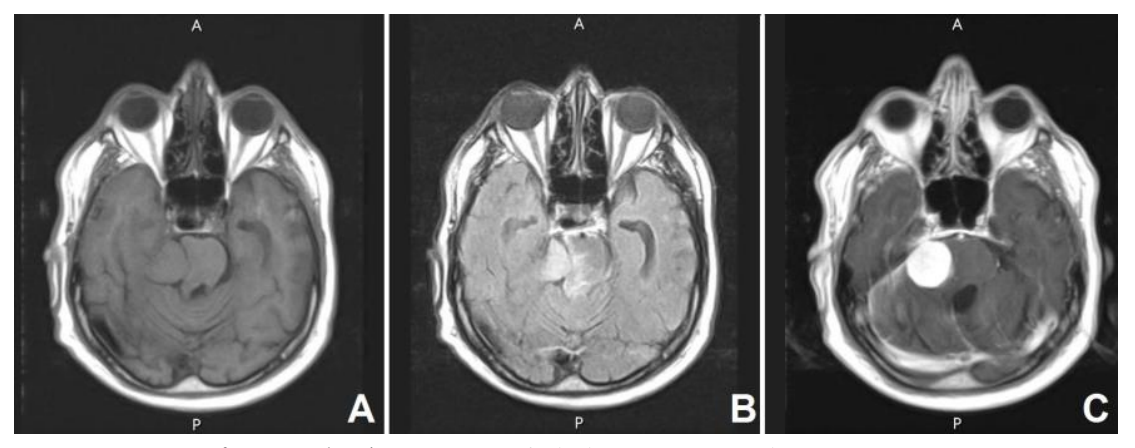

Figura 1: A.Corte axial de TC - ponderação T1; B.Corte axial de TC - FLAIR;

C.Corte axial de TC - T1 pós contraste (gadolíneo).
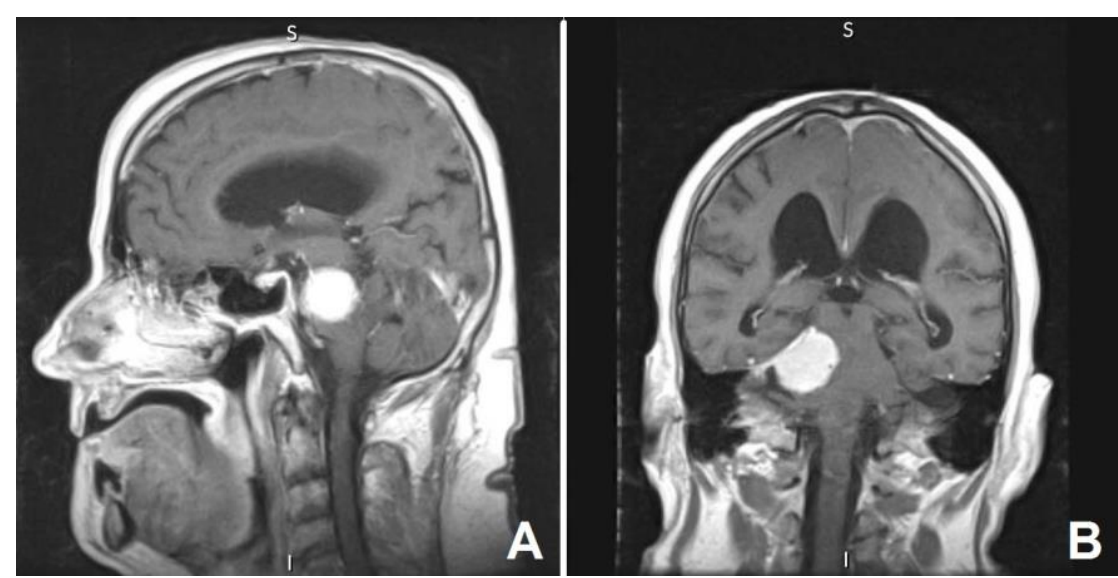

Figura 2: A. Corte sagital de TC - T1 pós gadolíneo; B. Corte coronal de TC - T1 pós gadolíneo.

Neste mesmo período, o paciente começou a relatar náuseas ao andar de carro, episódios de engasgos durante a alimentação, lapsos de memória e suas quedas, na maioria das vezes, estavam associadas a quadros de vertigens. Além disso, a perda da resposta motora dos membros inferiores ficou mais evidente.

Tal sintomatologia pode ser justificada, uma vez que a formação nodular diagnosticada na RM exercia efeito compressivo no tronco encefálico (ponte) e pedúnculo cerebelar, além de compressão do IV ventrículo, com dilatação do III ventrículo e dos ventrículos laterais.

A RM mostrou, ainda, que a formação nodular tem efeito expansivo sobre o tronco cerebral, pedúnculo cerebral e porções periféricas do parênquima cerebral, associado ás alterações nos sinais semelhantes a edema vasogênico destas regiões. O feito expansivo provocou colabamento parcial do 
IV ventrículo e dilatação dos ventrículos cerebrais supratentoriais.

O tempo decorrido desde os primeiros sinais e sintomas até o diagnóstico definitivo da lesão foi de 4 meses. $\mathrm{E}$, imediatamente, após a definição de meningioma na tenda cerebelar foi realizada a ressecção completa da lesão e o material coletado foi encaminhado para análise anatomopatológica.

Histopatologicamente, verificou-se a proliferação de células meníngeas sustentadas por fibras colágenas recortando o tumor em múltiplas direções e em distribuição irregular. Em um maior detalhe, observaram-se as células meníngeas neoplásicas dispostas em ninhos em forma de verticilo arredondados (Figura 3A), as quais apresentavam discreta atipia nuclear e alguns núcleos claros de aspecto lavado (Figura 3B).
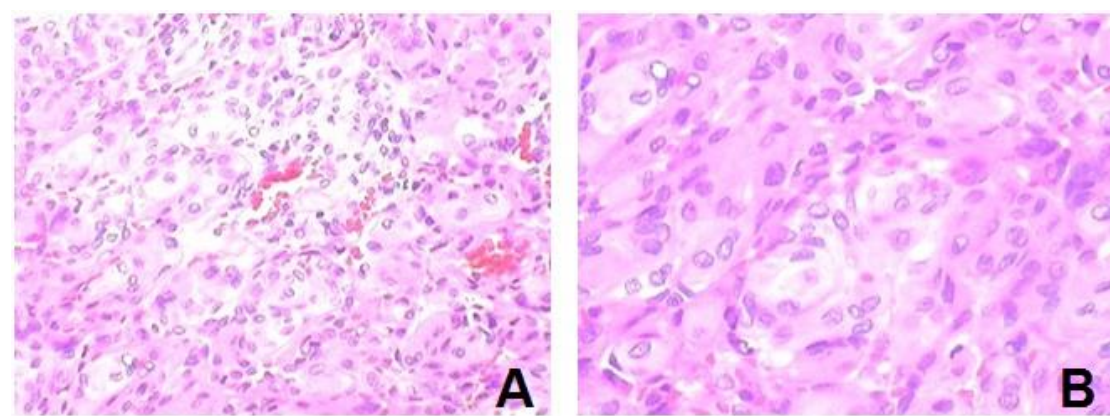

Figura 3: A. Presença de células meníngeas neoplásicas formando verticilos arredondados. B: Presença de atipia nuclear discreta e núcleos claros.

A ressecção cirúrgica foi realizada com o paciente em decúbito ventral, com cabeça fixada no suporte de 3 pontos e apoiado em coxins de espuma. Primeiramente, realizou-se a tricotomia na região occipital, seguida de degermação, antissepsia e colocação de campos estéreis.

A incisão foi do tipo paramediana occipital direita, que permitiu a exposição da região mastoide e occipital, e posterior acesso suboccipital lateral direito (craniectomia). Realizou-se uma abertura circular da dura-máter que possibilitou a visualização de uma herniação do hemisfério cerebelar, nesta foi coloca o microscópio operatório para análise do tecido.

Houve a drenagem de líquor da cisterna magna para descompressão, permitindo o encontro da massa neoplásica na tenda do cerebelo e base do crânio (rochedo temporal), sendo este um tumor sangrativo. A desintegração tumoral da base do crânio foi feita com cautério bipolar ("debulking" tumoral interno).

A dissecção microcirúrgica circunferencial tumoral ocorreu com descolamento do tumor do tronco cerebral e dos nervos cranianos baixos, possibilitando a ressecção tumoral completa localizada na fossa posterior do cérebro. A hemostasia foi feita com cautério bipolar e surgicel.

A dissecção foi finalizada com o fechamento da duramáter e colocação de um dreno aspirativo extradural seguido pelo fechamento dos planos cirúrgicos. Uma nova degermação e a antissepsia da área foram realizadas antes do fechamento com curativo estéril. O procedimento terminou sem intercorrências. No entanto, no pós-operatório, o paciente apresentou complicações sistêmicas e foi a óbito. Desfecho que é raro em casos de meningioma tratado com ressecção cirúrgica completa.

\section{DISCUSSÃO}

Apesar dos autores Costa et al. $^{3}$ afirmarem que o meningioma intracraniano primário é mais comum em mulheres com idade média de 45 anos, o caso apresentado ocorreu em um paciente do sexo masculino na sétima década de vida, mostrando a importância da investigação clínica em ambos os sexos, independente da idade.

As manifestações clínicas como cefaleia, náusea e vômito, apresentadas pelo paciente, também foram relatadas por Lima et al. ${ }^{5}$, estando associadas ao quadro clínico de hipertensão intracraniana causada pelo aumento do líquor em diagnósticos tardios. Casos sintomáticos, como o que foi relatado, mostram a importância de uma investigação mais específica e minuciosa, a fim de acelerar o diagnóstico e, consequentemente, o tratamento, contribuindo para um melhor prognóstico do paciente. No entanto, se o paciente for assintomático, como alertaram Pamir et al. ${ }^{10}$, o achado acidental do tumor deve ser avaliado pelo histórico clínico e o acompanhamento do meningioma deve ser realizado periodicamente por meio de exames complementares.

Os exames complementares mais indicados para o diagnóstico de tumores intracranianos de acordo com Pimentel $^{1}$ são a TC e a RM, porém para o diagnóstico do caso relatado foi utilizada, exclusivamente, a RM que mostrou uma formação nodular homogênea de imagem semelhante à cauda dural na tenda cerebelar e presença de edema vasogênico perilesional. Tal formação nodular exercia efeito compressivo no tronco encefálico (ponte) e pedúnculo cerebelar, além de compressão do IV ventrículo, com dilatação do III ventrículo e dos ventrículos laterais. A possibilidade de microangiopatia foi considerada no diagnóstico diferencial, mas a análise histopatológica definiu o diagnóstico de meningioma.

A intervenção terapêutica do caso relatado foi a ressecção completa, uma vez que a topografia e as dimensões da lesão verificadas na RM possibilitaram a instituição do tratamento neurocirúrgico como descrito por Al-Mefty et al. ${ }^{11}$.

A ressecção cirúrgica completa da lesão foi realizada sem qualquer intercorrência fortalecendo a conduta adequada. No entanto, no pós-operatório o paciente apresentou complicações sistêmicas e foi a óbito. Dessa forma, pode-se corroborar com o trabalho descrito por Jesús et al. ${ }^{9}$ no qual relatou que o bom prognóstico do paciente depende, também, da higidez do organismo do paciente, não só da acessibilidade e da ressecção completa do tumor, como observado por Costa Jr et al. ${ }^{3}$ e Al-Mefty et al. ${ }^{11}$.

E, embora, a maior causa de morte pós-operatória seja devido à progressão tumoral por inadequada resposta ao tratamento, como indicaram Lima et al. ${ }^{5}$, o caso relatado apresentou como causa de óbito: idade avançada, hipertensão intracraniana e más condições clínicas pósoperatórias, estando de acordo com Sacko et al. ${ }^{12}$.

\section{CONCLUSÃO}

O caso relatado enfatizou os aspectos clínicos, imaginológicos e histopatológicos do meningioma com a finalidade de ampliar o conhecimento do médico generalista, favorecendo o diagnóstico e o tratamento da doença. Mediante tal relato, pode-se concluir que a investigação clínica é essencial mesmo em pacientes fora do grupo de maior incidência da lesão, sendo que o conhecimento das principais características da doença facilita o diagnóstico precoce e, consequentemente, antecipa o tratamento adequado, resultando no melhor prognóstico do paciente.

\section{REFERÊNCIAS}

1. Pimentel J. Meningiomas: presente e futuro. Acta Med Port. 1993; 6(5):199-207. 
2. Velasquez CC, Costa HA, Passos PRC, Ribeiro SS, Mendonça WJP, Queiroz LLC. Análise dos óbitos por neoplasia pulmonar em São Luís - MA. Rev Pesq Saúde. 2011; 12(2):44-7.

3. Costa Jr LB, Morais JV, Lemos S. Meningioma com transformação rabdóide: relato de caso. Arq NeuroPsiquiatr. 2003; 61(2-A):277-80.

4. Cambruzzi E, Zettler CG, Zettler EW, Jotz GP, Grudzinski M, Pedrini J. Perfil imunoistoquímico de metástases do sistema nervoso central. Rev AMRIGS. 2009; 53(4):382-7.

5. Lima ER, Resende JA, Ibiapina CC, Oliveira BM Análise de sobrevida de pacientes portadores de tumores do sistema nervoso central. Rev Med Minas Gerais. 2015; 25(Supl 6):S10-6.

6. Castro FD, Reis F, Guerras JGG. Lesões expansivas intraventriculares à ressonância magnética: ensaio iconográfico - parte 1. Radiol Bras. 2014; 47(3):176-81.

7. Valenzuela R, Ebensperguer E. Base de craneo: anatomia y patologia tumoral Revision conceptual. Rev chil radiol. 2002;8(4):170-7.

8. Shogan P, Banks KP, Brown S. AJR teaching file: Intraventricular mass. AJR Am J Roentgenol. 2007; 189(6 Suppl):S55-7.

9. De Jesús O, Sekhar LN, Parikh HK, Wright DC, Wagner DP. Long-term follow-up of patients with meningiomas involving the cavernous sinus: recurrence, progression, and quality of life. Neurosurgery. 1996;39(5):915-20.

10. Pamir MN, Black McL, Fahlbusch R. Meningiomas: a comprehensive text. Philadelphia:Saunders/Elsevier; 2010.

11. Al-Mefty O, DeMonte F, McDermott MF. Al-Mefty's meningiomas. New York:Thieme Medical; 2011.

12. Sacko O, Sesay M, Roux FE, Riem T, Grenier B, Liguoro D, et al. Intracranial meningioma surgery in the ninth decade of life. Neurosurgery. 2007; 61(5):950-4.

\section{CONFLITO DE INTERESSES}

Os autores declaram não haver conflitos de interesse.

\section{AUTOR PARA CORRESPONDÊNCIA}

Luciana Estevam Simonato

luciana.simonato@universidadebrasil.edu.br

Submetido em 20/03/2017

Aceito em 02/05/2017 